\title{
Extubating in the operating room after adult cardiac surgery safely improves outcomes and lowers costs
}

\author{
Vinay Badhwar, MD, Stephen Esper, MD, Maria Brooks, PhD, Suresh Mulukutla, MD, \\ Regina Hardison, MS, Demetri Mallios, BS, Danny Chu, MD, Lawrence Wei, MD, and \\ Kathirvel Subramaniam, MD
}

\begin{abstract}
Objective: Prolonged intubation has been implicated in the poor outcomes after adult cardiac surgery. Accelerated postoperative extubation has been a quality focus, but operating room (OR) extubation after cardiopulmonary bypass is rare. We examined the outcomes and direct costs of protocolized OR extubation versus early postoperative intensive care unit (ICU) extubation after nonemergency open cardiac surgery.
\end{abstract}

\begin{abstract}
Methods: From January 2012 to June 2013, 652 consecutive patients who had undergone various cardiac operations, including redo and multivalve operations, were extubated within 12 hours, 165 in the OR. The OR extubation patients were propensity matched from multivariable logistic regression to derive 106 matched pairs for OR extubation versus extubation $<12$ hours (group 1 ) and 98 independently matched pairs for OR extubation versus extubation $<6$ hours (group 2).

Results: OR versus ICU extubation conveyed significant reductions in ICU hours (26.3, interquartile range [IQR], 22.0-31.0; vs 29.0, IQR, 25.0-51.0; $P=.001$, for group 1; 27.0, IQR, 22.0-32.0; vs 29.0, IQR, 25.0$54.0 ; P=.0002$, for group 2) and postoperative length of stay (5 days, IQR, 4-6; vs 6 days, IQR, 5-7; $P=.0008$, for group $1 ; 5$ days, IQR, 4-6; vs 6 days, IQR, 4-7; $P=.0002$, for group 2 ) but did not affect the reintubation rate $(1.9 \%$ [2 of 106 ] vs $0.0 \%$ [0 of 106], $P=.5$, group $1 ; 3.1 \%$ [3 of 98 ] vs $2.0 \%$ [2 of 98 ], $P=1.0$, group 2). OR versus ICU extubation conferred a $>20 \%$ cost reduction from surgery completion to discharge (\$3055, IQR, \$2576-\$3964; vs \$3977, IQR, \$3028-\$4947; $P=.0007$, group 1; $\$ 3025$, IQR, \$2598-\$3965, vs \$3877, IQR, \$2998-\$5458; $P=.007$, group 2).
\end{abstract}

Conclusions: After cardiac surgery, OR extubation is safe and might provide improvement in length of stay and cost compared with early postoperative ICU extubation. (J Thorac Cardiovasc Surg 2014;148:3101-9)

Supplemental material is available online.

Prolonged intubation after adult cardiac surgery has been implicated in protracted intensive care unit (ICU) times and postoperative length of stay (LOS). ${ }^{1,2}$ Defined as mechanical ventilation $>24$ hours, the hospital rates of postoperative prolonged intubation are currently tracked as a National Quality Forum performance indicator owing to the known associations with greater rates of nosocomial pneumonia, 30-day operative mortality, and poor

\footnotetext{
From the Heart and Vascular Institute, University of Pittsburgh Medical Center, Pittsburgh, $\mathrm{Pa}$

Disclosures: Authors have nothing to disclose with regard to commercial support.

Read at the 94th Annual Meeting of The American Association for Thoracic Surgery, Toronto, Ontario Canada, April 26-30, 2014.

Received for publication April 5, 2014; revisions received July 4, 2014; accepted for publication July 7, 2014; available ahead of print Aug 28, 2014.

Address for reprints: Vinay Badhwar, MD, Division of Cardiac Surgery, Heart and Vascular Institute, University of Pittsburgh Medical Center, 200 Lothrop St, Suite C-721, Pittsburgh, PA 15213 (E-mail: badhwarv@upmc.edu). $0022-5223 / \$ 36.00$

Copyright (c) 2014 by The American Association for Thoracic Surgery http://dx.doi.org/10.1016/j.jtcvs.2014.07.037
}

outcomes. $^{3-5}$ Early extubation after cardiac surgery has been linked to safe improvements in LOS, resource usage, and mortality. ${ }^{5-7}$ The timelines of outcome-enhancing "fast-track" cardiac anesthesia management remain incompletely defined, ranging from $<12$ hours to $<6$ hours. ${ }^{8-10}$ Although expedient extubation after cardiac operations remains controversial, ${ }^{11-13}$ protocolized pathways to decrease ventilator time have shown incremental timerelated improvement in outcomes without increasing complications such as reintubation. ${ }^{14-16}$ Experience with safe operating room (OR) extubation after adult cardiac surgery remains rare and currently confined to highly selected patients and operation types. ${ }^{16-18}$ Therefore, our objective was to assess the effect of OR extubation in a propensity-matched comparison to early postoperative ICU extubation on the outcomes and cost after all nonemergency open cardiac operations.

\section{METHODS \\ Patient Population}

From January 1, 2012, to June 30, 2013, 888 patients underwent adult cardiac surgery at the Presbyterian University Hospital of the University of Pittsburgh Medical Center. Our study cohort of 652 consecutive patients receiving all forms of open cardiac operations who had been extubated within 12 hours was formed after excluding 197 operations defined as 


$$
\begin{aligned}
& \text { Abbreviations and Acronyms } \\
& \text { CABG }=\text { coronary artery bypass grafting } \\
& \text { ICU }=\text { intensive care unit } \\
& \text { IQR }=\text { interquartile range } \\
& \text { LOS }=\text { length of stay } \\
& \text { OR }=\text { operating room }
\end{aligned}
$$

emergent, those performed for acute bacterial endocarditis, transcatheter aortic valve replacement, transplantation, or requiring deep hypothermic circulatory arrest, shock, or ventricular assist device implantation, and 39 patients requiring ventilation $>12$ hours. The inclusion and exclusion patient flow diagram is illustrated in Figure 1. OR extubation was achieved in 165 patients. Of the 487 patients extubated in the ICU within $<12$ hours, 356 were extubated within $<6$ hours. Preoperative baseline demographic and clinical comorbid risk variables and operative procedural variables were recorded and analyzed with the approval of the institutional review board of the University of Pittsburgh Medical Center.

\section{Anesthesia and Extubation Protocol}

All patients in the present study underwent a similar protocolized approach to cardiac anesthesia induction, maintenance, and emergence. After anesthetic informed consent was obtained, a limited dose of 1 to 2 mg midazolam premedication was administered in the preoperative holding area or on arrival to the operating room. Although the most common surgical approach was sternotomy, in selected thoracotomy patients, we used intrathecal morphine for postoperative analgesia that was administered before anesthetic induction. For these cases, the patients were placed in sitting position, and 0.2 to $0.5 \mathrm{mg}$ of intrathecal morphine was administered after confirmation of cerebrospinal fluid aspiration through a 25 guage spinal needle. The patients undergoing sternotomy or those with altered coagulation profiles, severe back pain, previous back surgery, or refusal of regional anesthesia did not receive an intrathecal injection. After application of the monitors, all patients were placed in the supine position, and an invasive arterial line was inserted before induction. An initial limited dose of a short-acting opioid, $<100 \mu \mathrm{g}$ fentanyl, was administered during induction. Additional dosing of the opioid analgesic was individualized at the clinical discretion of the cardiac anesthesiologist. Only fentanyl or fentanyl plus hydromorphone was used for analgesia, with the total dose of fentanyl limited to 250 to $500 \mu \mathrm{g}$ and hydromorphone to 1 to $2 \mathrm{mg}$. Hydromorphone was generally not administered if intrathecal morphine was used. After induction of general anesthesia with propofol or etomidate, a volatile agent, usually isoflurane, was used for maintenance of anesthesia before the institution of cardiopulmonary bypass. No additional benzodiazepines were given, and the depth of anesthesia was maintained only with inhalational agents. The anesthetic depth was monitored to a bispectral index target of 40 to 60 throughout the operation. After separation from cardiopulmonary bypass, a short-acting volatile agent such as desflurane or sevoflurane was substituted for isoflurane. Throughout the procedure, the neuromuscular blockade was monitored very closely, with the anticipation of blockade reversal at procedure completion. Intravenous acetaminophen was often administered to further augment the analgesia. After completion of each procedure, the neuromuscular blockade was reversed, and the patients were extubated once the typical criteria had been fulfilled. The extubation criteria included normothermia, stable hemodynamics, the absence of mediastinal bleeding, adequate muscle strength and respiratory effort with a tidal volume of $>5 \mathrm{~mL} / \mathrm{kg}$, and a fully awake patient capable of following commands. The patients who did not fulfill the criteria in the OR were given temporary propofol or dexmedetomidine sedation only for ICU transport. On arrival at the ICU and the initial ICU assessment, the sedation was discontinued and weaning from mechanical ventilation was initiated according to a preset nursing and respiratory therapist protocol. Once the criteria had been fulfilled, the patients were extubated, and the time was recorded in the electronic medical record. Postoperative analgesia after extubation was provided with acetaminophen, limited intravenous morphine to a maximum of $10 \mathrm{mg} / \mathrm{h}$, or oral oxycodone, as appropriate, to a visual analog pain scale score of $<4$.

\section{Propensity Score and Statistical Analyses}

The baseline demographic, clinical, and procedural characteristics were compared between the 165 patients extubated in the OR and the 487 patients extubated in the ICU within $<12$ hours. All preoperative and operative variables from our institutional Society of Thoracic Surgeons, version 2.73 , adult cardiac surgery database were used without exclusion to create propensity scores for OR extubation from multivariable logistic regression to create 2 matched groups: OR extubation versus ICU extubation within $<12$ hours (group 1) and OR extubation versus ICU extubation within $<6$ hours (group 2). Within each group, the propensity score represented the probability that a patient would undergo OR extubation instead of ICU extubation. Two independent multivariable logistic regression models were created, and the corresponding propensity scores for each patient were calculated. Stepwise regression methods using an $\alpha$ of 0.20 threshold were used to select and retain the variables in the propensity logistic regression model. Hosmer-Lemeshow statistics were used to evaluate the goodness of fit of the propensity model. The baseline characteristics for the

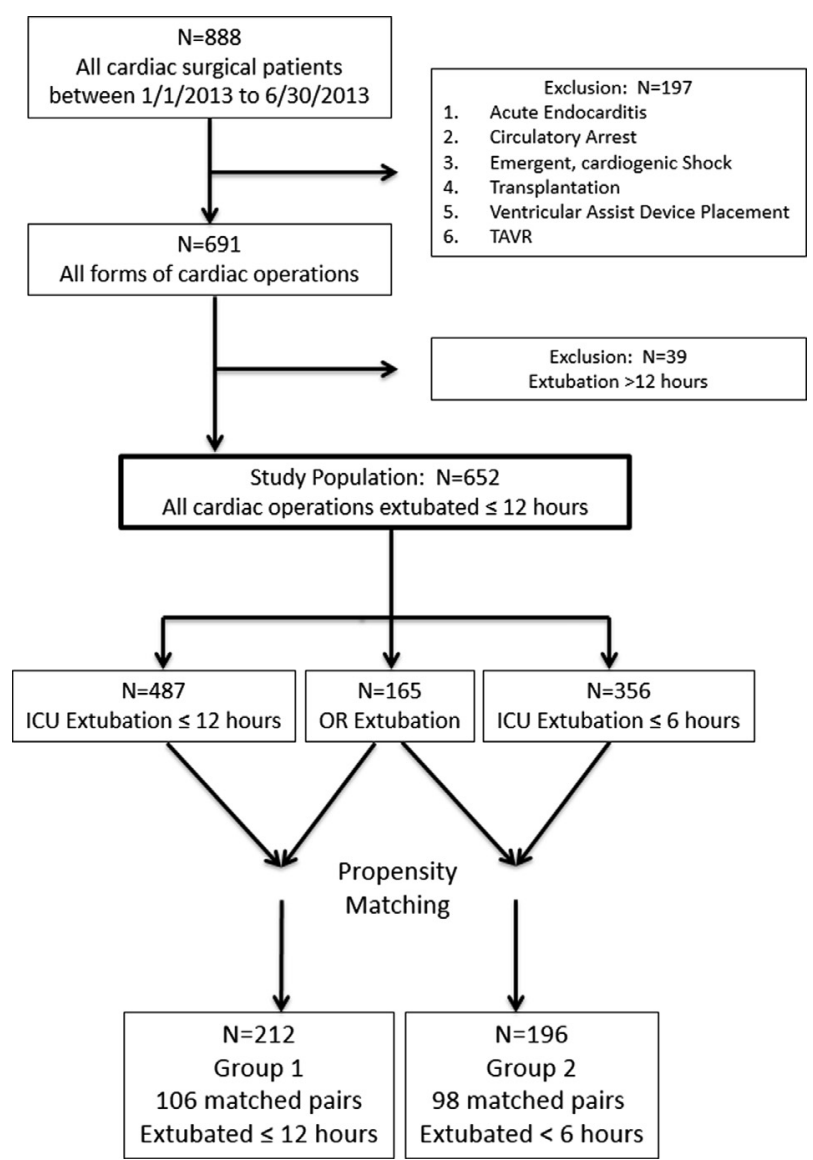

FIGURE 1. Flow diagram displaying the sequence of patient inclusion, classification of extubation in the operating room, and outside the operating room, and identification of the matched pair groups. $I C U$, Intensive care unit; TAVR, transcatheter aortic valve replacement. 
matched patient sets were compared to ensure adequate balance. The model demonstrated reasonable goodness of fit (Hosmer-Lemeshow statistic, $8.48,8 \mathrm{df}, P=.39$ ), and the propensity scores among the 652 patients representing the predicted probability of extubation ranged from 0.002 to 0.96. From the calculated propensity scores, patients undergoing OR extubation were randomly sequenced and matched $1: 1$ without replacement with the ICU extubation patients using a caliper distance of 0.10 to derive 106 matched pairs in group $1(\mathrm{n}=212)$ and 98 independently matched pairs in group $2(\mathrm{n}=196)$.

In addition to the clinical outcomes between the groups and extubation status, the primary endpoints were the assessment of ICU hours, postoperative LOS in days, reintubation rate, and the rate of direct home discharge without skilled nursing. Direct cost accounting for each patient was examined by a review of the institutional financial records from surgery completion to discharge.

The data are presented as the mean \pm standard deviation or median and interquartile range (IQR) for continuous variables and as proportions for categorical variables. Wilcoxon rank-sum tests were used to compare continuous variables and the chi-square test or Fischer's exact test was used, when appropriate, for dichotomous variables. The analyses were performed using Statistical Analysis Systems, version 9.2, and R (SAS Institute Inc, Cary, NC).

\section{RESULTS \\ Patient Characteristics}

Before propensity matching of the full cohort, the OR extubation patients were more likely to have undergone elective surgery, were slightly younger, leaner, and more commonly male, and had fewer symptoms of heart failure or angina than the patients extubated within $<12$ hours in the ICU. Similarly, only 40\% (66 of 165) of the OR extubation patients had undergone a full sternotomy compared with $90.6 \%$ (441 of 487 ) of those extubated in the ICU $(P<.0001)$. However, although the unmatched OR extubation patients were more likely to have undergone minimally invasive right thoracotomy (38.8\% [64 of 165]), 63.6\% (105 of 165) underwent open valve operations compared with only $39.2 \%$ (191 of 487 ) of the ICU extubation population. After multivariable propensity score regression modeling, the factors independently associated with OR extubation were active smoking, a lower body mass index, unstable angina at presentation, and full sternotomy (Appendix Table E1). After even matching between groups 1 and 2, the previously highlighted differences in operation type, incision type, and elective status were eliminated in the matched data sets used for the remainder of the analyses (Table 1).

\section{Outcome for Propensity Score-Matched Sets}

The matched analyses of the study endpoints demonstrated that extubation in the OR resulted in similar clinical outcomes and safety parameters compared with early extubation in the ICU but with lower healthcare usage and cost (Table 2). No difference was found in the total operative time between OR and ICU extubation (median, 374 minutes [IQR, 316-441] vs median, 378 minutes [IQR, 331-428]; $P=.32$ for group 1; median, 379 minutes [IQR, 316-439] vs median, 384 minutes [IQR, 339-432]; $P=.21$ for group 2). A small numeric difference was found in the minutes from "drapes off" to "wheels out" between patients extubated in the OR and patients extubated in the ICU (median, 22 minutes [IQR, 19-31] vs median, 20 [IQR, 15-25]; $P=.0035$, group 1; median, 23 minutes [IQR, 19-31] vs median, 20 minutes [IQR, 15-25]; $P=.0024$, group 2). Extubation in the OR versus early postoperatively in the ICU did not significantly affect the composite frequency of any postoperative complication $(27.4 \%$ [29 of 106] vs $38.7 \%$ [41 of 106], $P=.09$, group $1 ; 27.6 \%$ [27 of 98] vs $40.8 \%$ [40 of 98], $P=.06$, group 2; Figure $2, A$ ) or the reintubation rate $(1.9 \%$ [ 2 of 106 ] vs $0.0 \%$ [0 of 106], $P=.5$, group $1 ; 3.1 \%$ [3 of 98] vs $2.0 \%$ [2 of 98], $P=1.0$, group 2). Similarly, the extubation location had no bearing on the incidence of major morbidity or mortality (Table 2). However, in the matched samples, the patients extubated in the OR had significant reductions in postoperative ICU hours compared with those extubated early in the ICU (median, 26.3 [IQR, 22.0-31.0] vs median, 29.0 [IQR, 25.0-51.0], $P=.001$, group 1; median, 27.0 [IQR, 22.0-32.0] vs median, 29.0 [IQR, 25.0-54.0], $P=.0002$, group 2), with similar reductions seen in surgery completion to discharge LOS (median, 5 days [IQR, 4-6] vs median, 6 days [IQR, 5-7], $P=.0008$, group 1 ; median, 5 days [IQR, 4-6] vs median, 6 days [IQR, 4-7], $P=.0002$, group 2; Figure 2, $B$ ). In a subset analysis excluding all minimally invasive operations, the findings were identical (Table 3). Moreover, extubation in the OR led to significantly greater rates of direct to home discharge without the need for skilled nursing interval rehabilitation $(88.7 \%$ [94 of 106] vs $72.6 \%$ [77 of 106], $P=.0009$, group $1 ; 88.8 \%$ [87 of 98] vs 73.5\% [72 of 98], $P=.01$, group 2; Figure 2, A). An overall $>20 \%$ cost reduction from surgery completion to discharge was associated with OR extubation compared with early ICU extubation (median, \$3055 [IQR, \$2576-\$3964] vs median, \$3977 [IQR, \$3028-\$4947], $P=.0007$, group 1; median, \$3025 [IQR, \$2598-\$3965] vs median, \$3877 [IQR, $\$ 2998-\$ 5458], P=.007$, group 2).

\section{DISCUSSION}

We investigated protocolized OR extubation after all nonemergency open cardiac operations, including redo operations and multivalve procedures, performed by way of sternotomy and nonsternotomy approaches, and compared the outcomes and cost to propensity-matched cases of early ICU extubation. The key finding of the present study was that OR extubation can be safely performed without augmented risk of reintubation and confers significant benefits over early postoperative or "fast-track" extubation.

\section{Case Selection and Outcomes}

Avoidance of prolonged mechanical ventilation after adult cardiac surgery is a known international quality metric 
TABLE 1. Baseline and operative characteristics of total population and propensity-matched groups by extubation status

\begin{tabular}{|c|c|c|c|c|c|c|c|c|c|}
\hline \multirow[b]{2}{*}{ Characteristic } & \multicolumn{3}{|c|}{ Full patient population $(n=652)$} & \multicolumn{3}{|c|}{ Group $1(n=212)$} & \multicolumn{3}{|c|}{ Group $2(n=196)$} \\
\hline & $\begin{array}{c}\text { Extubated } \\
\text { in OR } \\
(\mathrm{n}=165) \\
\end{array}$ & $\begin{array}{c}\text { Extubated } \\
\leq 12 \mathrm{~h} \\
(\mathrm{n}=\mathbf{4 8 7}) \\
\end{array}$ & $P$ value & $\begin{array}{c}\text { Extubated } \\
\text { in OR } \\
(\mathrm{n}=106) \\
\end{array}$ & $\begin{array}{c}\text { Extubated } \\
\leq 12 \mathrm{~h} \\
(\mathrm{n}=106)\end{array}$ & $P$ value & $\begin{array}{c}\text { Extubated } \\
\text { in OR } \\
(\mathbf{n}=98) \\
\end{array}$ & $\begin{array}{c}\text { Extubated } \\
<6 \mathbf{~ h} \\
(\mathbf{n}=98) \\
\end{array}$ & $P$ value \\
\hline Age (y) & $57.8 \pm 12.8$ & $65.2 \pm 12.0$ & $<.0001$ & $60.6 \pm 12$ & $59.8 \pm 14.1$ & .6564 & $60.6 \pm 12.3$ & $61.5 \pm 14.4$ & 6400 \\
\hline Female sex & $31.5(52)$ & $36.6(178)$ & .2421 & $29.2(31)$ & $31.1(33)$ & .7648 & $30.6(30)$ & $31.6(31)$ & .8774 \\
\hline BMI $\left(\mathrm{kg} / \mathrm{m}^{2}\right)$ & $27.3 \pm 5.1$ & $29.3 \pm 6.3$ & $<.0001$ & $28.2 \pm 5.5$ & $27.6 \pm 5.3$ & .3849 & $27.8 \pm 5.5$ & $27.9 \pm 5.3$ & .9909 \\
\hline $\mathrm{CHF}$ & $57.6(95)$ & $43.3(211)$ & .0015 & $46.2(49)$ & $43.4(46)$ & .6786 & $42.9(42)$ & $48.0(47)$ & 4732 \\
\hline Class I & $7.4(7)$ & $0.5(1)$ & $<.0001$ & 0 & 0 & .8937 & 0 & 0 & .2971 \\
\hline Class II & $35.8(34)$ & $22.3(47)$ & & $22.4(11)$ & $26.1(12)$ & & $19.0(8)$ & $31.9(15)$ & \\
\hline Class III & $48.4(46)$ & $59.2(125)$ & & $63.3(31)$ & $58.7(27)$ & & $64.3(27)$ & $48.9(23)$ & \\
\hline Class IV & $8.4(8)$ & $18.0(38)$ & & $14.3(7)$ & $15.2(7)$ & & $16.7(7)$ & $19.1(9)$ & \\
\hline Diabetes & $23.0(38)$ & 38.4 (187) & .0003 & $30.2(32)$ & $30.2(32)$ & 1.0000 & $31.6(31)$ & $28.6(28)$ & .6404 \\
\hline Last $\mathrm{HbA1c}$ level & $5.7(5.4-6.0)$ & $5.9(5.5-6.5)$ & $<.0001$ & $5.8(5.4-6.2)$ & $5.7(5.5-6.3)$ & 6376 & $5.8(5.5-6.2)$ & $5.7(5.5-6.2)$ & .9688 \\
\hline Dialysis & $1.8(3)$ & $2.5(12)$ & .6325 & $2.8(3)$ & $0.9(1)$ & .3127 & $3.1(3)$ & $1.0(1)$ & .3123 \\
\hline Hypertension & 72.1 (119) & 89.7 (437) & $<.0001$ & $82.1(87)$ & $84.0(89)$ & .7145 & $80.6(79)$ & $86.7(85)$ & .2462 \\
\hline CVD & $12.1(20)$ & $19.5(95)$ & .0315 & $12.3(13)$ & $12.3(13)$ & 1.0000 & $14.3(14)$ & $21.4(21)$ & 1917 \\
\hline CVA & $3.0(5)$ & $7.8(38)$ & .0340 & $3.8(4)$ & $4.7(5)$ & .7334 & $4.1(4)$ & $8.2(8)$ & .2334 \\
\hline Chronic lung disease & & & .0007 & & & .0528 & & & .3068 \\
\hline Mild & $10.3(17)$ & $12.9(63)$ & & $14.2(15)$ & $6.6(7)$ & & $14.3(14)$ & $10.2(10)$ & \\
\hline Moderate & $5.5(9)$ & $9.9(48)$ & & $5.7(6)$ & $7.5(8)$ & & $7.1(7)$ & $9.2(9)$ & \\
\hline Severe & $0.6(1)$ & $7.6(37)$ & & $0.9(1)$ & $6.6(7)$ & & $1.0(1)$ & $5.1(5)$ & \\
\hline PVD & $10.9(18)$ & $24.6(120)$ & .0002 & $14.2(15)$ & $14.2(15)$ & 1.0000 & $16.3(16)$ & $16.3(16)$ & 1.0000 \\
\hline Immunosuppressive therapy & $4.8(8)$ & $5.3(26)$ & .8066 & $5.7(6)$ & $5.7(6)$ & 1.0000 & $7.1(7)$ & $6.1(6)$ & .7741 \\
\hline \multicolumn{10}{|l|}{ Risk factors } \\
\hline Current/recent smoker & $20.0(33)$ & $18.7(91)$ & .7101 & $22.6(24)$ & $25.5(27)$ & .6298 & $26.5(26)$ & $24.5(24)$ & .7431 \\
\hline Home oxygen & $0.6(1)$ & $3.9(19)$ & .0339 & $0.9(1)$ & $2.8(3)$ & .3127 & $1.0(1)$ & $3.1(3)$ & .3123 \\
\hline Inhaled bronchodilator & $9.1(15)$ & $17.7(86)$ & .0086 & $12.3(13)$ & $17.0(18)$ & .3311 & $13.3(13)$ & $12.2(12)$ & .8305 \\
\hline Pneumonia & $6.7(11)$ & $9.4(46)$ & .2747 & $9.4(10)$ & $6.6(7)$ & .4481 & $9.2(9)$ & $5.1(5)$ & .2673 \\
\hline Sleep apnea & $13.3(22)$ & $16.8(82)$ & .2880 & $17.0(18)$ & $15.1(16)$ & .7082 & $15.3(15)$ & $12.2(12)$ & .5341 \\
\hline Syncope & $4.8(8)$ & $5.5(27)$ & .7319 & $4.7(5)$ & $4.7(5)$ & 1.0000 & $3.1(3)$ & $7.1(7)$ & 1941 \\
\hline Carotid stenosis & $0.6(1)$ & $2.7(13)$ & .1140 & $0.9(1)$ & $2.8(3)$ & .3127 & $1.0(1)$ & $4.1(4)$ & .1741 \\
\hline Stroke or TIA & $8.5(14)$ & $9.7(47)$ & .6567 & $9.4(10)$ & $9.4(10)$ & 1.0000 & $10.2(10)$ & $12.2(12)$ & .6509 \\
\hline Liver disease & $3.0(5)$ & $8.6(42)$ & .0163 & $4.7(5)$ & $3.8(4)$ & .7334 & $3.1(3)$ & $4.1(4)$ & .7003 \\
\hline MELD score & $7.5(6.4-9.1)$ & $8.4(7.5-10.0)$ & $<.0001$ & $7.5(6.4-9.2)$ & $7.6(7.5-9.2)$ & .4128 & $7.5(6.4-9.2)$ & $7.8(6.4-9.7)$ & .1738 \\
\hline MELD score $>10$ & $13.9(23)$ & $24.9(121)$ & .0035 & $15.1(16)$ & $11.3(12)$ & .4171 & $15.3(15)$ & $20.4(20)$ & .3511 \\
\hline Last hematocrit & $40.2(37.0-43.0)$ & $38.0(34.0-41.1)$ & $<.0001$ & $39.0(36.2-42.6)$ & $39.2(37.0-42.2)$ & .9366 & $38.7(36.0-42.0)$ & $39.5(36.6-43.0)$ & 4116 \\
\hline Mediastinal radiotherapy & $0.6(1)$ & $3.9(19)$ & .0339 & $0.9(1)$ & $0.9(1)$ & 1.0000 & $1.0(1)$ & $3.1(3)$ & .3123 \\
\hline Albumin & $3.9(3.6-4.2)$ & $3.7(3.3-4.0)$ & $<.0001$ & $3.7(3.5-4.1)$ & $3.8(3.5-4.0)$ & .8327 & $3.7(3.5-4.0)$ & $3.8(3.5-4.1)$ & .2636 \\
\hline Previous CABG & $1.8(3)$ & $6.4(31)$ & .0239 & $2.8(3)$ & $3.8(4)$ & .7007 & $2.0(2)$ & $3.1(3)$ & .6505 \\
\hline Previous valve & $3.7(6)$ & $7.8(38)$ & .0675 & $5.7(6)$ & $6.6(7)$ & .7747 & $6.1(6)$ & $8.2(8)$ & .5791 \\
\hline \multicolumn{10}{|l|}{ Preoperative cardiac status } \\
\hline Previous MI & $17.6(29)$ & $33.5(163)$ & .0001 & $24.5(26)$ & $31.1(33)$ & .2834 & $24.5(24)$ & $25.5(25)$ & .8690 \\
\hline Angina class & & & .0046 & & & .9367 & & & .8426 \\
\hline CCS I & $1.2(2)$ & $0.6(3)$ & & $0.9(1)$ & $0.9(1)$ & & $1.0(1)$ & $1.0(1)$ & \\
\hline CCS II & $9.1(15)$ & $11.9(58)$ & & $11.3(12)$ & $10.4(11)$ & & $13.3(13)$ & $11.2(11)$ & \\
\hline CCS III & $13.3(22)$ & $17.0(83)$ & & $18.9(20)$ & $15.1(16)$ & & $17.3(17)$ & $12.2(12)$ & \\
\hline CCS IV & $7.3(12)$ & $16.6(81)$ & & $11.3(12)$ & $10.4(11)$ & & $11.2(11)$ & $13.3(13)$ & \\
\hline No symptoms & $69.1(114)$ & $53.8(262)$ & & $57.5(61)$ & $63.2(67)$ & & $57.1(56)$ & $62.2(61)$ & \\
\hline $\begin{array}{c}\text { Cardiac presentation at } \\
\text { admission }\end{array}$ & & & $<.0001$ & & & .7212 & & & .6259 \\
\hline No symptoms & $49.1(81)$ & $29.8(145)$ & & $34.0(36)$ & $31.1(33)$ & & $36.7(36)$ & $38.8(38)$ & \\
\hline NSTEMI & $4.2(7)$ & $10.9(53)$ & & $6.6(7)$ & $4.7(5)$ & & $6.1(6)$ & $11.2(11)$ & \\
\hline STEMI & $4.2(7)$ & $2.9(14)$ & & $6.6(7)$ & $2.8(3)$ & & $7.1(7)$ & $3.1(3)$ & \\
\hline Stable angina & $4.8(8)$ & $8.8(43)$ & & $6.6(7)$ & $6.6(7)$ & & $7.1(7)$ & $5.1(5)$ & \\
\hline Ischemia unlikely & $22.4(37)$ & $24.0(117)$ & & $26.4(28)$ & $30.2(32)$ & & $22.4(22)$ & $22.4(22)$ & \\
\hline Unstable angina & $15.2(25)$ & $23.6(115)$ & & $19.8(21)$ & $24.5(26)$ & & $20.4(20)$ & $19.4(19)$ & \\
\hline Proximal LAD disease $>70$ & $12.1(20)$ & $19.1(93)$ & .0408 & $17.0(18)$ & $12.3(13)$ & .3311 & $17.3(17)$ & $17.3(17)$ & 1.0000 \\
\hline Atrial fibrillation/flutter & $23.8(39)$ & $27.3(133)$ & .3753 & $22.6(24)$ & $21.7(23)$ & .8687 & $22.4(22)$ & $24.5(24)$ & .7361 \\
\hline Preoperative inotropes & $1.8(3)$ & $2.3(11)$ & .7358 & $2.8(3)$ & $2.8(3)$ & 1.0000 & $2.0(2)$ & $1.0(1)$ & .5607 \\
\hline Preoperative steroids & $0.6(1)$ & $1.4(7)$ & .4019 & 0.9 (1) & $1.9(2)$ & .5609 & $1.0(1)$ & $3.1(3)$ & .3123 \\
\hline
\end{tabular}




\begin{tabular}{|c|c|c|c|c|c|c|c|c|c|}
\hline \multirow[b]{2}{*}{ Characteristic } & \multicolumn{3}{|c|}{ Full patient population $(n=652)$} & \multicolumn{3}{|c|}{ Group $1(n=212)$} & \multicolumn{3}{|c|}{ Group $2(n=196)$} \\
\hline & $\begin{array}{l}\text { Extubated } \\
\text { in OR } \\
(\mathbf{n}=\mathbf{1 6 5})\end{array}$ & $\begin{array}{c}\text { Extubated } \\
\quad \leq 12 \mathrm{~h} \\
(\mathrm{n}=487)\end{array}$ & $P$ value & $\begin{array}{c}\text { Extubated } \\
\text { in OR } \\
(\mathbf{n}=106)\end{array}$ & $\begin{array}{c}\text { Extubated } \\
\leq 12 \mathrm{~h} \\
(\mathrm{n}=106)\end{array}$ & $P$ value & $\begin{array}{c}\text { Extubated } \\
\text { in OR } \\
(\mathbf{n}=98)\end{array}$ & $\begin{array}{c}\text { Extubated } \\
\quad<6 \mathbf{h} \\
(\mathrm{n}=98)\end{array}$ & $P$ value \\
\hline $\mathrm{EF}(\%)$ & $53.3 \pm 10.0$ & $51.2 \pm 13.3$ & .0361 & $52.8 \pm 9.9$ & $51.6 \pm 14.0$ & .4734 & $53.0 \pm 9.3$ & $52.1 \pm 13.4$ & .5609 \\
\hline \multicolumn{10}{|l|}{ Procedural information } \\
\hline Procedure type & & & $<.0001$ & & & .1117 & & & .0897 \\
\hline CABG & $25.5(42)$ & $37.8(184)$ & & $35.8(38)$ & $29.2(31)$ & & $37.8(37)$ & $30.6(30)$ & \\
\hline CABG + other & $0.0(0)$ & $1.6(8)$ & & $0.0(0)$ & $0.9(1)$ & & $0.0(0)$ & $2.0(2)$ & \\
\hline CABG + valve & $1.2(2)$ & $14.8(72)$ & & $1.9(2)$ & $10.4(11)$ & & $2.0(2)$ & $6.1(6)$ & \\
\hline CABG + valve + other & $0.6(1)$ & $4.1(20)$ & & $0.9(1)$ & $0.0(0)$ & & $1.0(1)$ & $3.1(30$ & \\
\hline Other & $9.1(15)$ & $2.5(12)$ & & $7.5(8)$ & $3.8(4)$ & & $9.2(9)$ & $2.0(2)$ & \\
\hline Valve & $44.8(74)$ & $23.0(112)$ & & $37.7(40)$ & $39.6(42)$ & & $35.7(35)$ & $36.7(36)$ & \\
\hline Valve + other & $18.8(31)$ & $16.2(79)$ & & $16.0(17)$ & $16.0(17)$ & & $14.3(14)$ & 19.4 (19) & \\
\hline Status & & & $<.0001$ & & & .4435 & & & .7543 \\
\hline Elective & $80.6(133)$ & $56.1(273)$ & & $69.8(74)$ & $74.5(79)$ & & $71.4(70)$ & $69.4(68)$ & \\
\hline Urgent & $19.4(32)$ & $43.9(214)$ & & $30.2(32)$ & $25.5(27)$ & & $28.6(28)$ & $30.6(30)$ & \\
\hline Operation incidence & & & .0232 & & & .2565 & & & .3317 \\
\hline First cardiac operation & $94.5(156)$ & $86.0(419)$ & & $92.5(98)$ & $89.6(95)$ & & $91.8(90)$ & $85.7(84)$ & \\
\hline First reoperation & $4.2(7)$ & $12.3(60)$ & & $5.7(6)$ & $9.4(10)$ & & $6.1(6)$ & $12.2(12)$ & \\
\hline Second reoperation & $1.2(2)$ & $1.2(6)$ & & $1.9(2)$ & $0.0(0)$ & & $2.0(2)$ & $2.0(2)$ & \\
\hline Third reoperation & $0.0(0)$ & $0.4(2)$ & & $0.0(0)$ & $0.9(1)$ & & 0 & 0 & \\
\hline Operative approach & & & $<.0001$ & & & .0754 & & & .0105 \\
\hline Full sternotomy & $40.0(66)$ & $90.6(441)$ & & $61.3(65)$ & $64.2(68)$ & & $67.3(66)$ & $66.3(65)$ & \\
\hline Partial upper sternotomy & $11.5(19)$ & $5.1(25)$ & & $10.4(11)$ & $19.8(21)$ & & $7.1(7)$ & $19.4(19)$ & \\
\hline Mini-right thoracotomy & $38.8(64)$ & $2.9(14)$ & & $17.0(18)$ & $10.4(11)$ & & $15.3(15)$ & $11.2(11)$ & \\
\hline Mini-left thoracotomy & $7.3(12)$ & $0.6(3)$ & & $7.5(8)$ & $1.9(2)$ & & $7.1(7)$ & $0.0(0)$ & \\
\hline Minimally invasive other & $2.4(4)$ & $0.8(4)$ & & $3.8(4)$ & $3.8(4)$ & & $3.1(3)$ & $3.1(3)$ & \\
\hline $\mathrm{CPB}$ time & $131.6(70.1)$ & $129.5(61.2)$ & .73 & $120.5(69.9)$ & $142.4(66.2)$ & .02 & $117.1(67.0)$ & $134.2(66.1)$ & .074 \\
\hline
\end{tabular}

because prolonged mechanical ventilation has been linked to poor outcomes and mortality. ${ }^{3-5}$ Early or "fast-track" extubation protocols have been identified as a method to safely improve outcomes and reduce the postoperative ICU time without the need for reintubation. ${ }^{6-8,15,16}$ Early extubation within $<6$ or 12 hours has been predominantly studied after coronary artery bypass grafting (CABG), with many studies highlighting off-pump techniques. ${ }^{7-9,15-18}$ In our quaternary center, the use of off-pump CABG has been a rare and selective practice. Beginning in mid-2011, we applied the principles of protocolized early extubation strategies similar to those of Fitch and colleagues ${ }^{15}$ to improve our postoperative extubation times. Observing that anesthesia technique variability had an affect on extubation, we implemented a standardized anesthetic protocol for all cardiac surgical procedures. This enabled a comprehensive strategy to mitigate prolonged ventilation across all operation types. OR extubation was initially applied to those undergoing minimally invasive right thoracotomy mitral valve repair operations; however, by January 2012, this experience was applied to all nonemergency operations, regardless of the incision type, procedure complexity, or redo status. Although the merits of early or "fast-track" extubation are clear, any additional advantage potentially conferred by OR extubation is unknown. Therefore, our clinical quality improvement objective was to apply the principles of preoperative and intraoperative protocolized care pathways of extubation to all operations and assess the potential benefits of OR extubation. In our study, the procedures addressed the breadth of conventional onpump cardiac operations, including multivalve surgery, concomitant open Cox-maze IV procedures, and valve plus CABG. Nearly $10 \%$ of matched patients had had a preoperative stroke and a Model for End-Stage Liver Disease score $>10$ and had undergone redo operations. One quarter were in New York Heart Association class III-IV heart failure and had undergone concomitant open multiprocedure valve operations. With an average body mass index of $28 \mathrm{~kg} / \mathrm{m}^{2}$, our patients approached the obesity definition of $30 \mathrm{~kg} / \mathrm{m}^{2}$, and $15 \%$ had sleep apnea.

The practice of OR extubation is not without controversy, with some detractors evoking a catecholamine stress response or hypoxia-related injury related to premature extubation that could result in myocardial compromise and cardiopulmonary embarrassment. ${ }^{1-13}$ The argument has been that the incidence of bleeding and airway 
TABLE 2. Postoperative healthcare usage and clinical outcomes for the propensity matched groups by extubation status

\begin{tabular}{|c|c|c|c|c|c|c|}
\hline \multirow[b]{2}{*}{ Outcome } & \multicolumn{3}{|c|}{ Group $1(n=212)$} & \multicolumn{3}{|c|}{ Group $2(n=196)$} \\
\hline & $\begin{array}{c}\text { Extubated } \\
\text { in OR } \\
(\mathrm{n}=\mathbf{1 0 6}) \\
\end{array}$ & $\begin{array}{c}\text { Extubated } \\
\leq 12 \mathrm{~h} \\
(\mathrm{n}=106)\end{array}$ & $P$ value & $\begin{array}{c}\text { Extubated } \\
\text { in OR } \\
(\mathbf{n}=\mathbf{9 8}) \\
\end{array}$ & $\begin{array}{c}\text { Extubated } \\
<6 \mathrm{~h} \\
(\mathrm{n}=98) \\
\end{array}$ & $P$ value \\
\hline \multicolumn{7}{|l|}{ OR time (min) } \\
\hline Total OR time (patient in-patient out) & $374(316-441)$ & $378(331-428)$ & .32 & $379(316-439)$ & $384(339-432)$ & .21 \\
\hline Surgery completion to patient out & $22(19-30)$ & $20(15-25)$ & .0035 & $23(19-31)$ & $20(15-25)$ & .0024 \\
\hline \multicolumn{7}{|l|}{ LOS } \\
\hline Total ICU (h) & $26.0(22.0-30.0)$ & $30.5(25.0-51.0)$ & .0004 & $26.8(22.0-31.0)$ & $30.0(25.0-69.0)$ & $<.0001$ \\
\hline Surgery completion to discharge (d) & $5.0(4.0-6.0)$ & $6.0(5.0-7.0)$ & .0008 & $5.0(4.0-6.0)$ & $6.0(4.0-7.0)$ & .0005 \\
\hline \multicolumn{7}{|l|}{ Cost } \\
\hline ICU (US \$) & & & .0005 & & & .0272 \\
\hline Mean \pm SD & $1566 \pm 933$ & $2268 \pm 1726$ & & $1605 \pm 1073$ & $2997 \pm 6075$ & \\
\hline Median (IQR) & $1298(1088-1521)$ & $1508(1236-2510)$ & & $1335(1088-1570)$ & $1484(1236-3413)$ & \\
\hline Step down (US \$) & & & .0761 & & & .0130 \\
\hline Mean \pm SD & $1898 \pm 1013$ & $2208 \pm 1705$ & & $1881 \pm 999$ & $2413 \pm 2109$ & \\
\hline Median (IQR) & $1505(1323-2284)$ & $1870(1380-2431)$ & & $1476(1309-2222)$ & $1841(1380-2749)$ & \\
\hline Surgery completion to discharge (total US \$) & & & .0007 & & & .0073 \\
\hline Mean $\pm \mathrm{SD}$ & $3464 \pm 1430$ & $4477 \pm 2694$ & & $3486 \pm 1442$ & $5410 \pm 6843$ & \\
\hline Median (IQR) & $3055(2575-3964)$ & $3976(3028-4947)$ & & $3025(2598-3964)$ & $3876(2998-5456)$ & \\
\hline \multicolumn{7}{|l|}{ Clinical outcome } \\
\hline Reintubation & $1.9(2)$ & $0.0(0)$ & .50 & $3.1(3)$ & $2.0(2)$ & .6505 \\
\hline Stroke/transient TIA & $0.9(1)$ & $0.9(1)$ & 1.0 & $1.0(1)$ & $0.0(0)$ & .3161 \\
\hline Reoperation, bleeding & $1.9(2)$ & $0.0(0)$ & .50 & $2.0(2)$ & $0.0(0)$ & .1552 \\
\hline Pneumonia & $0.0(0)$ & $0.0(0)$ & - & $0.0(0)$ & $2.0(2)$ & .1552 \\
\hline Renal failure & $0.0(0)$ & $1.9(2)$ & .50 & $0.0(0)$ & $1.0(1)$ & .3161 \\
\hline Atrial fibrillation & $24.5(26)$ & $28.3(30)$ & .62 & $23.5(23)$ & $31.6(31)$ & .2009 \\
\hline Presence of any complication & $27.4(29)$ & $38.7(41)$ & .096 & $27.6(27)$ & $40.8(40)$ & .0503 \\
\hline Discharged to home & $88.7(94)$ & $72.6(77)$ & .0009 & $88.8(87)$ & $73.5(72)$ & .0062 \\
\hline 30-d mortality & $0.0(0)$ & $0.9(1)$ & 1.0 & $0.0(0)$ & $0.0(0)$ & - \\
\hline
\end{tabular}

Data presented as $\%(\mathrm{n})$, mean $\pm \mathrm{SD}$, or median (IQR). OR, Operating room; $L O S$, length of stay; ICU, intensive care unit; $S D$, standard deviation; IQR, interquartile range; TIA, transient ischemic attack.

complications are high in the immediate postoperative period and delaying extubation for $\geq 6$ hours will help to stabilize those issues before weaning the patient from ventilatory support. In a nonrandomized study of 100 CABG patients, Montes and colleagues ${ }^{13}$ noted that compared with ICU extubation, patients extubated in the OR had no difference in ICU or hospital LOS but incurred a reintubation rate of $8 \%$ and postoperative myocardial infarction rate of $3 \%$. The results of that early experience might be explained by the anesthetic technique used. The thiopental and pancuronium used in their study are associated with prolonged recovery. The use of pancuronium has also been associated with significant residual neuromuscular paralysis or recurarization and might play a role in the greater reintubation rates. ${ }^{20}$ The use of benzodiazepinebased techniques has similarly been associated with delayed extubation, impaired mental function, and prolonged ICU time. ${ }^{21}$ In our center, thiopental and pancuronium have been replaced by low doses of propofol, etomidate, fentanyl, and shorter acting rocuronium. The practice of highdose benzodiazepine cardiac anesthesia has been replaced by low-dose midazolam restricted to anxiolysis at the onset of anesthesia, avoiding repeat "top-up" doses, even during rewarming on cardiopulmonary bypass. Inhalation anesthesia is used and titrated to keep the bispectral index at 40 to 60 to maintain the anesthesia depth without compromising prompt recovery. In our study, we found that not only was OR extubation safe in terms of no difference in the low reintubation rates compared with ICU extubation, but also OR extubation was associated with significant improvements in patient mobilization, pulmonary toilet, and a return to physiologic baseline as manifested by the shorter ICU times and reductions in LOS, without the sequelae of an increased incidence of complications.

Earlier experiences with immediate OR extubation were enhanced by the use of high thoracic epidural anesthesia and focused on isolated CABG or isolated aortic valve replacement. ${ }^{16,18,19}$ Although we used a single intrathecal (spinal) opioid injection for postoperative analgesia in only a few of the minimally invasive thoracotomy operations in the present series (18.4\% [39 of 212] in group 1, 16.8\% [33 of 196] in group 2), no patient received epidural anesthesia. Most patients examined in our study underwent a sternotomy operation performed 


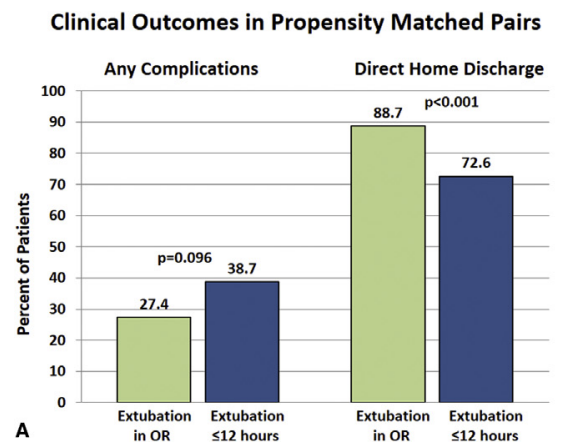

Length of Stay Outcomes in Propensity Matched Pairs
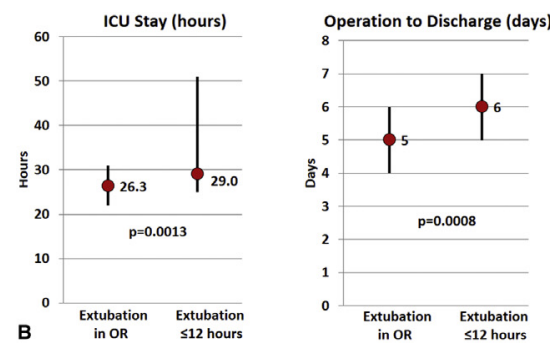

FIGURE 2. A comparison of clinical and length of stay outcomes between the group 1 matched set of patients who were extubated in the operating room $(\mathrm{n}=106)$ and patients who were extubated outside the operating room $\leq 12$ hours $(\mathrm{n}=106)$. A, The percentage of patients Left, who had any complication and Right, who were discharged from the hospital directly to their home. $P$ values were determined from chi-square statistics from conditional logistic regression models stratified by the matched pairs. B, The median (red circle) and interquartile ranges (black lines) for the length of stay Left, in the intensive care unit and Right, from surgery to hospital discharge. $P$ values were based on Wilcoxon signed rank tests stratified by the matched pairs.

on-pump and received conventionally balanced anesthesia. Although the present study was propensity matched to include the procedure and incision type, a separate subset analysis excluding minimally invasive operations further confirmed the identical outcome reductions associated with OR extubation.

\section{Resource Usage}

The pursuit of high-quality and cost-efficient care is the touchstone for all cardiac surgical programs in the current era of value-based healthcare. By understanding the variability of common cardiac operations, an opportunity exists for improvement in both quality and resource usage. $^{22,23}$ The complications that occur after our operative procedures can be among the most costly, not only to our patients, but also to our healthcare system. ${ }^{24}$ Methods to mitigate complications and reduce the LOS after common cardiac operations serve to provide value and quality dividends. Streamlining the process and procedures to decrease the extubation time after cardiac surgery is one such initiative.

Early or "fast-track" cardiac anesthesia protocols have shown promising cost effectiveness with selected operations.
In a randomized controlled trial of isolated $\mathrm{CABG}$ patients, Cheng and colleagues ${ }^{25}$ showed that substantial cost savings could be realized when patients were extubated within 6 hours, helping to set national benchmarks for CABG. In a Cochrane review of 25 trials and 4118 patients, fast track cardiac surgery did not increase patient morbidity or mortality or reduce overall hospitalization; however, low-dose narcotic techniques and time-based extubation protocols within 12 hours were responsible for substantial reductions in the ICU stay. ${ }^{26}$ Chamchad and colleagues $^{27}$ used CABG registry data to confirm that early or even OR extubation could be performed with a reintubation rate of $<1 \%$ and the hospital stay could be reduced by 0.8 day.

In our study, we sought to determine whether OR extubation could confer enhanced value compared with established early "fast-track" intervals for CABG while applying this strategy for all forms of open cardiac operations. Because the data on extubation for 12 to 24 hours after cardiac surgery appears well established, we focused our analysis on comparing OR extubation to propensitymatched patients extubated within either $<12$ hours or $<6$ hours. We compared the interval from surgery completion to OR departure and found that OR extubation could be

TABLE 3. Postoperative healthcare usage and clinical outcomes for the propensity matched groups by extubation status—without minimally invasive surgery

\begin{tabular}{|c|c|c|c|c|c|c|}
\hline \multirow[b]{2}{*}{ Outcome } & \multicolumn{3}{|c|}{ Group $1(n=198)$} & \multicolumn{3}{|c|}{ Group $2(n=184)$} \\
\hline & $\begin{array}{c}\text { Extubated } \\
\text { in } \mathrm{OR}(\mathbf{n}=99)\end{array}$ & $\begin{array}{c}\text { Extubated } \\
\leq 12 \mathrm{~h}(\mathrm{n}=99)\end{array}$ & $P$ value & $\begin{array}{c}\text { Extubated } \\
\text { in } \mathrm{OR}(\mathrm{n}=92)\end{array}$ & $\begin{array}{c}\begin{array}{c}\text { Extubated } \\
<6 \mathrm{~h}(\mathrm{n}=92)\end{array}\end{array}$ & $P$ value \\
\hline Total ICU stay (h) & & & .0055 & & & .0008 \\
\hline Median & 26.0 & 29 & & 27.0 & 29.5 & \\
\hline IQR & $22.0-31.0$ & $25.0-50.0$ & & $22.0-31.5$ & $25.0-70.0$ & \\
\hline Surgery completion to discharge (d) & & & .0035 & & & .0003 \\
\hline Median & 5.0 & 6.0 & & 5.0 & 6.0 & \\
\hline IQR & $4.0-6.0$ & $5.0-7.0$ & & $4.0-6.0$ & $5.0-7.0$ & \\
\hline
\end{tabular}

$O R$, Operating room; $I C U$, intensive care unit; $I Q R$, interquartile range. 
accomplished efficiently without clinically or fiscally significant delays in OR time (Table 2). OR extubation was performed safely without an increase in morbidity, mortality, or reintubation across a variety of complex cardiac operations. Although OR extubation appeared to clearly show a trend toward a decreased overall complication rate, the difference did not reach statistical significance. We found that OR extubation after all forms on nonemergency cardiac operations provided enhanced value through a $20 \%$ postoperative cost reduction and improvements in the length of ICU stay, postoperative hospitalization, and direct-tohome healthy discharge.

Enhancements in surgical and anesthesia techniques and protocol-driven postoperative critical care management are enablers for team-based quality care improvement in cardiac surgery. Extubation in the OR should no longer be considered unacceptable after low-risk or, even, moderate-risk cardiac surgery. Extubation of awakened patients in the OR can offer several advantages, such as avoidance of the excessive sympathetic discharge associated with endotracheal stimulation, better recovery of vascular tone, and, potentially, less need for catecholamine support and improvements in physiologic venous return and related end-organ perfusion through nonmechanical spontaneous respiration. Reducing the need for postoperative sedatives and narcotics can also enhance the normal return to gastrointestinal and pulmonary function, thus, minimizing the risk of related complications. Enhancing the clinical cooperation and communication among the anesthesia, cardiac surgery, and critical care personnel through extubation protocols is one method of achieving lasting outcome improvement and value in cardiac surgery.

\section{Study Limitations}

The present study was observational and retrospective and variables other than what were included in the analysis could have been present and could have affected the results. Although propensity matching was done to avoid the potential for selection bias in our study, this method of statistical adjustment cannot account for unobserved variables that could have influenced the decision and timing to extubate a patient in the OR. Although the anesthesia technique was protocolized, individual anesthesiologists adjusted the type and dose of the drugs and the timing of their administration according to their clinical judgment. This might have influenced some of the differences in the patient recovery profiles. The effect of OR extubation on the dose changes of inotropic, vasopressor, and pain medications was not evaluated in the present study. Our study was not adequately powered to detect the difference in complications considering the low event rates for outcomes, such as myocardial infarction, renal failure, stroke, and 30-day mortality. Geographical distance and family support was not recorded; thus, any influence these might have had on direct to home discharge is not known. The present study of a well-matched limited patient volume cannot replace the inherent value of a prospective randomized controlled clinical trial.

\section{CONCLUSIONS}

The present study has provided evidence for the safety and efficacy of OR extubation after adult cardiac operations. Programmatic transitioning from early postoperative extubation to immediate OR extubation in low- and moderate-risk cardiac surgical patients could provide cost-effective outcome improvement.

\section{References}

1. Trouillet JL, Combes A, Vaissier E, Luyt CE, Quattara A, Pavie A, et al. Prolonged mechanical ventilation after cardiac surgery: outcome and predictors. $J$ Thorac Cardiovasc Surg. 2009;138:948-53.

2. Cheng DC, Karski J, Peniston C, Asokumar B, Raveendran G, Carroll J, et al. Morbidity outcome in early versus conventional tracheal extubation after coronary artery bypass grafting: a prospective randomized controlled trial. $J$ Thorac Cardiovasc Surg. 1996;112:755-64.

3. Reddy SLC, Grayson AD, Griffiths EM, Pullan DM, Rashid A. Logistic risk model for prolonged ventilation after adult cardiac surgery. Ann Thorac Surg. 2007;84:528-36.

4. O'Brien S, Edwards FH, Ferraris VA, Haan CK, Rich JB, Peterson ED, et al. Quality measurement in adult cardiac surgery. Part 2: statistical considerations in composite measure scoring and provider rating. Ann Thorac Surg. 2007;83: S13-26.

5. Hillis DL, Smith PK, Anderson JL, Bittl JA, Bridges CR, Byrne JG, et al. 2011 ACCF/AHA guideline for coronary artery bypass graft surgery: a report of the American College of Cardiology Foundation/American Heart Association task force on practice guidelines. Circulation. 2011;124:e652-735.

6. Reis J, Mota JC, Ponce P, Costa-Pereira A, Guerreiro M. Early extubation does not increase complication rates after coronary artery bypass graft surgery with cardiopulmonary bypass. Eur J Cardiothorac Surg. 2002;21:1026-30.

7. Silbert BS, Santamaria JD, O'Brien JL, Blyth CM, Kelly WJ, Molnar RR. Early extubation following coronary artery bypass surgery: a prospective randomized controlled trial. The Fast Track Cardiac Care Team. Chest. 1998;113:1481-8.

8. Oxelbark S, Bengtsson L, Eggersen M, Kopp J, Pedersen J, Sanchez R. Fast track as a routine for open heart surgery. Eur J Cardiothorac Surg. 2001;19:460-3.

9. Alhan C, Toraman F, Karabulut EH, Tarcan S, Dagdelen S, Eren N, et al. Fa0073t track recovery of high risk coronary bypass surgery patients. Eur J Cardiothorac Surg. 2003;23:678-83.

10. Myles PS, Daly DJ, Djaiani G, Lee A, Cheng DC. A systematic review of the safety and effectiveness of fast-track cardiac anesthesia. Anesthesiology. 2003; 99:982-7.

11. Sullivan BL. Con: early extubation in the operating room following cardiac surgery. Semin Cardiothorac Vasc Anesth. 2012;16:187-9.

12. Siliciano D. Con: early extubation is not preferable to late extubation in patients undergoing cardiac surgery. J Cardiothorac Vasc Anesth. 1992;6:494-8.

13. Montes FR, Sanchez SI, Giraldo JC, Rincon IE, Vanegas MV, Charris H. The lack of benefit of tracheal extubation in the operating room after coronary artery bypass surgery. Anesth Analg. 2000;91:776-80.

14. Guller U, Anstrom KJ, Holman WL, Allman RM, Sansom M, Petersen ED. Outcomes of early extubation after bypass surgery in the elderly. Ann Thorac Surg. 2004; 77:781-8.

15. Fitch ZW, Debesa O, Ohkuma R, Duquaine D, Steppan J, Schneider EB, et al. A protocol-driven approach to early extubation after heart surgery. J Thorac Cardiovasc Surg. 2014;147:1344-50.

16. Royse CF, Royse AG, Soeding PF. Routine immediate extubation after cardiac operation: a review of our first 100 patients. Ann Thorac Surg. 1999;68:1326-9.

17. Edgerton JR, Herbert MA, Prince SL, Horswell JL, Michelson L, Magee MJ, et al. Reduced atrial fibrillation in patients immediately extubated after offpump coronary artery bypass grafting. Ann Thorac Surg. 2006;81:2121-6.

18. Straka Z, Brucek P, Vanek T, Votava J, Widimsky P. Routine immediate extubation for off-pump coronary artery bypass grafting without thoracic epidural analgesia. Ann Thorac Surg. 2002;74:1544-7. 
19. Hemmerling TM, Le N, Olivier JF, Choiniere JL, Basile F, Prieto I. Immediate extubation after aortic valve surgery using high thoracic epidural analgesia or opioid-based analgesia. J Cardiothorac Vasc Anesth. 2005;19:176-81.

20. Murphy GS, Szokol JW, Marymont JH, Vender JS, Avram MJ, Rosengart TK, et al. Recovery of neuromuscular function after cardiac surgery: pancuronium versus rocuronium. Anesth Analg. 2003:96:1301-7.

21. Dowd NP, Karski JM, Cheung DC, Gajula S, Seneviratne P, Carroll-Munro JA, et al. Fast-track cardiac anesthesia in the elderly: effect of two different anesthetic techniques on mental recovery. Br J Anesth. 2001;86:68-76.

22. Kilic A, Shah AS, Conte JV, Mandal K, Baumgartner WA, Cameron DE, et al. Understanding variability in hospital-specific costs of coronary artery bypass grafting represents an opportunity for standardizing care and improving resource use. J Thorac Cardiovasc Surg. 2014;147:109-16.

23. Chen JC. Western Thoracic Surgical Association 2013 presidential address: winning the HITECH challenge. J Thorac Cardiovasc Surg. 2013;146:1006-15.

24. Ahmed EO, Butler R, Novick RJ. Failure-to-rescue rate as a measure of quality care in a cardiac surgical recovery unit: a five-year study. Ann Thorac Surg. 2014; 97:147-52.

25. Cheng DC, Karski J, Peniston C, Raveendran G, Asokumar B, Carroll J, et al. Early tracheal extubation after coronary artery bypass surgery reduces costs and improves resource use: a prospective, randomized, controlled trial. Anesthesiology. 1996;85:1300-10.

26. Hawkes CA, Dhileepan S, Foxcroft DR. Early extubation for adult cardiac surgical patients. Cochrane Database Syst Rev. 2003;4:CD003587.

27. Chamchad D, Horrow JC, Nachamchik L, Sutter FP, Samuels LE, Trace CL, et al. The impact of immediate extubation in the operating room after cardiac surgery on intensive care and hospital lengths of stay. J Cardiothorac Vasc Anesth. 2010; $24: 780-4$.

\section{Discussion}

Dr Glenn Whitman (Baltimore, $M d$ ). Dr Badhwar, as you and I have already spoken this past week, I think this is a tremendous study and extremely provocative. First, I have a comment. The propensity analysis was exhaustive, but it is hard to imagine that a group that is extubated in the OR can be compared with a group that required 12 hours to be extubated, and only possibly to a group that required $<6$ hours. It is hard to imagine why the ICU required 12 hours to extubate a group matched to the intraoperative extubation group, unless the groups were different.

The second point I wanted to make was that despite the propensity analysis that I had the opportunity to read-and it really was exhaustive; my congratulations. I think it would be comforting to see the Society of Thoracic Surgeons risk profile for the patients in the compared groups, to determine whether those data were comparable.

Finally, the data you presented does not show closure time to out-of-room time, which arguably could be significantly longer in the group extubated intraoperatively. All of us recognize that as the most expensive time in the entire hospitalization of a patient.
Your report is extremely provocative, and if in fact patient satisfaction significantly benefits by intraoperative extubation, it might be worth it just for that.

Dr Badhwar. Thank you for your kind comments and for your leadership and contributions in this field.

The grouping of $<12$ hours and $<6$ hours was done predominantly to answer questions in the published data on the definition of "fast tracking." Of the subgroup of 487 patients who were not extubated in the OR, 356 of those actually required $<6$ hours. So really this was a parallel examination of all extubation occurring within $<12$ hours but focused to $<6$ hours, as you very appropriately noted.

In terms of the propensity matching, it indeed incorporated every risk factor in our adult cardiac database without exclusion. One of our study limitations, as you point out, pertains to the OR decision-making regarding the clinical decision to extubate, which was fully outlined in the report.

We have done an analysis on the OR time expended to extubate. We examined the anesthesia record for times from "drapes off" to "wheels out" to define time spent from surgery end time to exiting the room. For patients in the $<6$-hour ICU extubation group, the median time was 20 minutes. For the OR extubation group, the median time was 23 minutes. Therefore, we importantly found that OR extubation could be performed with a fiscally negligible difference in OR time.

Thank you for your questions, and I agree with your comments on patient satisfaction, because this has been a substantial improvement.

Dr Daniel L. Miller (Marietta, $G a$ ). I enjoyed the presentation. How has your education of the patient preoperatively changed, because I think that is a very important part of all this, especially in getting patients out earlier and what they should expect in that first 6 to 12 hours. I would appreciate your expansion on that.

Dr Badhwar. Thank you, Dr Miller. The patients were extraordinarily satisfied, obviously, just as were their family and referring physicians. This has now become our routine for all patients undergoing all cardiac surgery. Starting first in the very early experience at the end of 2011 with minimally invasive surgery, now every single heart operation, regardless of the complexity or redo status, is prepared for OR extubation. The confidence rate for patients undergoing extubation in the OR we can somewhat predict from their clinical findings. We inform all patients to be prepared, to listen for the nurse's voice, to listen to the anesthesiologist's voice, to take deep breaths instead of small rapid breaths, and it has really enhanced our patient's experience with successful OR extubation.

Thank you for your question. 
APPENDIX TABLE E1. Propensity score logistic regression models

\begin{tabular}{|c|c|c|c|c|c|c|}
\hline \multirow[b]{2}{*}{ Covariate } & \multicolumn{3}{|c|}{$\begin{array}{l}\text { Propensity for extubation in OR } \\
\text { compared with extubation } \leq 12 \mathrm{~h}(\mathrm{n}=652)\end{array}$} & \multicolumn{3}{|c|}{$\begin{array}{c}\text { Propensity for extubation in OR } \\
\text { compared with extubation }<6 \mathrm{~h}(\mathrm{n}=521)\end{array}$} \\
\hline & $\begin{array}{c}\text { Regression } \\
\text { coefficient } \\
\text { estimate } \\
\end{array}$ & $\begin{array}{c}\text { Regression } \\
\text { coefficient } \\
\text { standard error }\end{array}$ & $P$ value & $\begin{array}{c}\text { Regression } \\
\text { coefficient } \\
\text { estimate } \\
\end{array}$ & $\begin{array}{c}\text { Regression } \\
\text { coefficient } \\
\text { standard error }\end{array}$ & $P$ value \\
\hline Age per year & -0.0206 & 0.00979 & .0351 & -0.0247 & 0.0103 & .0169 \\
\hline BMI per $1 \mathrm{U}$ increase & -0.0699 & 0.0220 & .0015 & -0.0562 & 0.0228 & .0136 \\
\hline Current cigarette smoking (yes vs no) & 0.9873 & 0.3399 & .0037 & 0.8234 & 0.3479 & .0179 \\
\hline Noncigarette tobacco use (yes vs no) & 1.3032 & 0.5677 & .0217 & 0.8466 & 0.5706 & .1369 \\
\hline CHF (yes vs no) & 0.8614 & 0.2895 & .0029 & 0.9113 & 0.2867 & .0015 \\
\hline PVD (yes vs no) & -0.7869 & 0.3461 & .0230 & -0.7923 & 0.3483 & .0229 \\
\hline Family history of CAD (yes vs no) & -0.5610 & 0.3087 & .0692 & -0.5025 & 0.3149 & .1106 \\
\hline Chronic lung disease (yes vs no) & -0.5276 & 0.3063 & .0850 & -0.6823 & 0.3116 & .0286 \\
\hline Liver disease (yes vs no) & -1.5560 & 0.6693 & .0201 & -1.9552 & 1.1717 & .0952 \\
\hline Previous heart failure (yes vs no) & -0.5977 & 0.3763 & .1122 & -0.6823 & 0.3116 & .0286 \\
\hline Mediastinal radiotherapy (yes vs no) & -1.6128 & 1.2048 & .1807 & -1.9552 & 1.1717 & .0952 \\
\hline Stroke/TIA (yes vs no) & 0.8971 & 0.4153 & .0308 & 0.9124 & 0.4292 & .0335 \\
\hline Last hematocrit per $1 \mathrm{U}$ increase & 0.0531 & 0.0235 & .0241 & 0.0419 & 0.0245 & .0871 \\
\hline Platelets per $1 \mathrm{U}$ increase & $-3.6 e-6$ & $2.198 \mathrm{e}-6$ & .0983 & $-3.25 e-6$ & $2.239 \mathrm{e}-6$ & .1472 \\
\hline MELD score per $1 \mathrm{U}$ increase & -0.0999 & 0.0446 & .0250 & -0.1393 & 0.0470 & .0031 \\
\hline Total albumin per $1 \mathrm{U}$ increase & - & - & - & 0.3156 & 0.2012 & .1167 \\
\hline $\begin{array}{l}\text { STEMI/NSTEMI/unstable angina presentation } \\
\text { (yes vs no) }\end{array}$ & 1.4184 & 0.4328 & .0010 & 0.7653 & 0.3162 & .0155 \\
\hline Full sternotomy (yes vs no) & -2.5372 & 0.2922 & $<.0001$ & -2.6042 & 0.2958 & $<.0001$ \\
\hline Urgent procedure (vs elective) & -0.7246 & 0.3116 & .0201 & - & - & - \\
\hline
\end{tabular}

$O R$, Operating room; $B M I$, body mass index; $C H F$, congestive heart failure; $P V D$, peripheral vascular disease; $C A D$, coronary artery disease; $T I A$, transient ischemic attack; MELD, Model for End-Stage Liver Disease score; NSTEMI, non-ST-segment elevation myocardial infarction; STEMI, ST-segment elevation myocardial infarction. 